\title{
Microcystin-leucine arginine blocks vasculogenesis and angiogenesis through impairing cytoskeleton and impeding endothelial cell migration by downregulating integrin-mediated Rho/ROCK signaling pathway
}

\section{Qilong Wang}

Chongqing University

Guoliang Chen

Chongqing University

Qian Zhang

Chongqing University

Mingxing Wang

Chongqing University

Guixue Wang

Chongqing University

Tingzhang Hu ( $\nabla$ tzhu2002@foxmail.com )

Chongqing University https://orcid.org/0000-0002-8755-4158

\section{Research Article}

Keywords: Cell migration, Cytoskeleton, Microcystin-leucine arginine, Vasculogenesis, Angiogenesis

Posted Date: May 5th, 2021

DOl: https://doi.org/10.21203/rs.3.rs-437480/v1

License: (c) (i) This work is licensed under a Creative Commons Attribution 4.0 International License. Read Full License

Version of Record: A version of this preprint was published at Environmental Science and Pollution Research on July 9th, 2021. See the published version at https://doi.org/10.1007/s11356-021-15337-9. 


\section{Abstract}

The main characteristic of eutrophication is cyanobacteria harmful algae blooms. Microcystin-leucine arginine (MC-LR) is considered to be the most toxic and most commonly secondary metabolite produced by cyanobacteria. It has been reported that MC-LR had potential vascular toxicity. However, the mechanism that MC-LR induced vascular toxicity is very limited and remain to be clarified. The aim of this study was to evaluate the toxic hazard towards the vasculogenesis and angiogenesis of MC-LR. Its effects on vasculogenesis, sprouting angiogenesis and endothelial cell tube formation were studied. The study showed that MC-LR exposure blocked vasculogenesis in zebrafish embryos, sprouting angiogenesis from rat aorta and tube formation of human umbilical vein endothelial cells (HUVECs). In addition, MC-LR exposure also induced the disruption of cytoskeletal structures and markedly inhibited endothelial cells (ECs) migration from caudal hematopoietic tissue in zebrafish and HUVEC migration. Western blot analysis showed that MC-LR exposure down-regulated the expressions of integrin $\beta 1, F A K$, Rho and ROCK. Combined with these results, MC-LR could induce disruption of cytoskeleton via downregulating integrin-mediated FAK/ROCK signaling pathway, leading to the inhibition of ECs migration, which finally blocked vasculogenesis and angiogenesis.

\section{Introduction}

Owing to climate change and irrational anthropogenic activities, eutrophication in freshwater, such as ponds, streams, swamps, lakes and rivers etc., and marine ecosystems has an increasing trend worldwide (O'Neil et al. 2012; Preece et al. 2017). The main characteristic of eutrophication is cyanobacteria harmful algae blooms (cyano-HABs). The overgrowth of harmful algae cause deterioration of water quality and emit an unpleasant smell, leading to the loss of recreational and property values of aquatic ecosystems (Zhang et al. 2021). In cyano-HABs, several cyanobacterial genera such as Anabaena, Microcystis, Nostoc and Planktothrix can produce toxic secondary metabolites (Dziga et al. 2013), which can inhibit the growth of other taxa and increase the competitive and defensive abilities (Liu et al. 2020). The existence of these toxins in waterbodies for recreation, drinking water and irrigation can pose serious human health risks and thus become an increasingly concerned public health topic (Preece et al. 2021).

In freshwater water, among the multifarious cyanobacteria (blue-green algae), microcystis aeruginosa is the most common genera producing microcystins. So far, a family of more than 200 different structurally monocyclic heptapeptide toxins, with microcystin-leucine arginine (MC-LR) considered to be the most toxic and most commonly encountered microcystin analogues (Puddick et al. 2014; Zhou et al. 2018). It has been reported that that MC-LR can damage the liver, kidneys, lungs, brain, heart, blood vessels, intestines, pancreatic islets, gonads, and reproductive system (Wang et al. 2019; Zhang et al. 2021). A large quantity of data have showed that MC-LR cause morphological injuries by inducing the abnormal condensation of microtubules, the shortening and loss of actin filaments as well as the reorganization of intermediate filaments, suggesting that MC-LR has cytoskeletal-specific effects containing reorganization of all three essential cytoskeletal structures (Wickstrom et al. 1995; Zhou et al. 2015). 
The vascular system is constructed and maintained by vasculogenesis and angiogenesis (Benson and Southgate 2021). Vasculogenesis is the development of a primary blood system via the de novo formation of blood vessels during embryogenesis, which is that endothelial precursor cells in the embryonic mesenchyme form an aggregate, and then mature into small, single-layered endothelial tubes (Kubis and Levy 2003; Semenza 2007). Angiogenesis is the formation of new blood vessels by sprouting or intussusception from pre-existing vessels, which is vital to a range of processes containing embryonic or fetal development, organ growth, wound tissue healing, skeletal development, pregnancy or during the menstrual cycle (Carmeliet 2005; Carmeliet and Jain 2011). Angiogenesis also is related to various kinds of pathological conditions, such as cancer, diabetic retinopathy, autoimmune disorders, arthritis, infectious diseases, and so on (Chung and Ferrara 2011). Sprouting angiogenesis is the expansion of existing vessels. Once the vessel has fully expanded, endothelial cells (ECs) proliferation and migration are inhibited by anti-angiogenic factors. Therefore, in the development of vascular networks, the proliferation and migration of ECs are critical processes, which are influenced by the activity of small Rho GTPases (Benson and Southgate 2021).

The cytoskeleton consists of three major structural elements: microfilaments, microtubules, and intermediate filaments, which plays a central role in a myriad of cellular processes such as the establishment and maintenance of cell architecture, cell shape, motility, growth, division, differentiation, apoptosis, and the transport or positioning of organelles (Ding et al. 2000; Henty et al. 2011). Contraction of the actin cytoskeleton is one necessary element of individual cell migration, which facilitates both forward movement of the cell body and detachment of the trailing edge (Schwarzbauer 1997).

Furthermore, contraction has been proposed to generate tension within three-dimensional matrices that conduces to lead migration of ECs toward one another to promote tube formation (Davis and Camarillo 1995). Thus, The perturbation of the cytoskeleton could involve not only direct inhibition of cell motility but also possibly interfere with guidance mechanisms for triggering cell-cell interaction networks.

Zebrafish (Danio rerio) is a frequently used as a general fish and vertebrate model for investigating chemical toxicity and mechanism, because its high fecundity, rapid development, a short life cycle, transparency of the eggs and embryos and easy to maintenance in the laboratory (Wang et al. 2020). Therefore, zebrafish embryos were used to evaluate vasculogenesis and angiogenesis. The rat aortic ring model has been proven to be the most useful method for the evaluation of the angiogenic process (Nicosia and Ottinetti 1990; Blacher et al. 2001).

The goal of the present study was to test whether MC-LR blocked vasculogenesis and angiogenesis by inhibiting EC migration. Toward this goal, studies were undertaken with the objective of evaluating the toxic effects of MC-LR on vessel networks in rat aorta rings, ECs migration and vasculogenesis in zebrafish, and migration and tube formation of human umbilical vein endothelial cells (HUVECs). In addition, actin cytoskeleton and Rho/ROCK signaling pathways in HUVEC progression and dissemination was examined.

\section{Materials And Methods}




\section{Rat aorta ring assay}

Adult healthy Sprague-Dawley (SD) rats specific pathogen-free (SPF) grade, weighing 200-220 g, were purchased from the Experimental Animal Center of Army Military Medical University (Chongqing, China). Rat aorta was obtained from a normal adult SD rats in accordance with the protocol approved by the ethical committee of the college of bioengineering of Chongqing University. All procedures were in compliance with the China Council on Animal Care and Use protocol.

On the day of experimentation, the adult SD rats were euthanized by an overdose of phenobarbital (50 $\mathrm{mg} / \mathrm{kg}$ body weight). After being rapidly dissected and isolated, the thoracic aorta placed in preheated (37 ${ }^{\circ} \mathrm{C}$ ) phosphate buffered saline (PBS). Following being carefully cleaned of adhering connective and fat tissues, the aorta was washed thrice with preheated PBS and cut into ring segments of $1 \mathrm{~mm}$ in length. Exposure of the gel in a solution of sulfo-SANPAH with a UV light source at 365 or $320 \mathrm{~nm}$ covalently links the sulfo-SANPAH to the polyacrylamide hydrogel. After adding an appropriate amount of rat tail tendon type I collagen (Solarbio, Beijing, China), the solution with the gel was incubated overnight at 37 ${ }^{\circ} \mathrm{C}$. Rat thoracic aortic rings were plated on the top of rat tail tendon type I collagen incubating in M199 medium from Gibco (Thermo Fisher Scientific, Waltham, MA, USA) containing 10\% fetal bovine serum (FBS; Gibco, California, USA), 50 ng/mL vascular endothelial growth factor 165 (VEGF 165), and designated concentrations of MC-LR (100 and $1000 \mathrm{nM})$ for 2 weeks, changing the spent medium with fresh medium every other day. Vascular sprouting from each ring was examined with an Olympus microscope (20x magnification; Olympus, Tokyo, Japan), and the captured images were analyzed by using NIH Scion Image software (Scion Corp., Frederick, MD).

\section{Breeding and exposure of zebrafish}

Adult zebrafish including $\mathrm{Tg}$ (flk1: EGFP) expressed enhanced green fluorescent protein (GFP) in ECs and $\mathrm{Tg}$ (fli1a: NGFP) expressed GFP in nucleus of ECs were provided by Prof. Anming Meng of Tsinghua University. Zebrafish were maintained and exposured according to the method described by Wang et al. (2019). Tg (flk1: EGFP) and Tg (fli1a: NGFP) embryos at 2 hours post fertilization (hpf) were collected and were placed into in $5 \mathrm{~mL}$ medium containing various concentrations of MC-LR (100 and $1000 \mathrm{nM})$. The $\mathrm{Tg}$ (flk1: EGFP) embryos were taken at $24 \mathrm{hpf}$ to evaluate vasculogenesis. The $\mathrm{Tg}$ (fli1a: NGFP) transgenic zebrafish line embryos were taken at $72 \mathrm{hpf}$ to evaluate the numbers of EC migration from caudal hematopoietic tissue (CHT). The embryos were examined using a stereoscopic microscope (Stemi 2000 C, Carl Zeiss, Jena, Germany) and analyzed by ImageJ software.

\section{EC tube formation assay}

Cell tube formation assay was performed as follows (Shi et al. 2017). Each well of the 96-well plate was coated with $80 \mu \mathrm{L} \mathrm{BD}$ matrigel (BD Biosciences, San Jose, CA) at $37^{\circ} \mathrm{C}$ for $1 \mathrm{~h}$. After starvation treatment of HUVECs in logarithmic phase, HUVECs were suspended in M199 medium containing $2 \%$ FBS and designated concentrations of MC-LR (100 and $1000 \mathrm{nM})$. Then, the HUVECs were plated onto the matrigel 
layer with a density of $1 \times 10^{5}$ cells/well and cultured with $5 \% \mathrm{CO}_{2}$ at $37^{\circ} \mathrm{C}$ for $12 \mathrm{~h}$. The tube networks were visualized by using an Olympus microscope.

\section{HUVEC migration assay}

Cell migration was measured adopting a monolayer scratch injury assay as described by Wang et al. (2020). Briefly, the HUVECs were seeded on glass chamber slides and cultured until confluence. Then, a uniform straight scratch was created in the confluent monolayer with a plastic Cell Scrapper (Corning, USA). The cell monolayers were washed gently and examined using an inverted microscope (Nikon Eclipse TE2000-Y system, Japan). All images were analyzed with the Image J software.

\section{cytoskeleton observation}

The cytoskeleton of HUVECs were assessed according to the method described by Wang et al. (2020). After being cultured with fresh cell medium containing the different concentrations of MC-LR $(1,10,100$ and $1000 \mathrm{nM}$ ) for $24 \mathrm{~h}$, the cytoskeleton were observed and images were captured using a confocal laserscanning microscope (CLSM; TCS SP8, Leica, Germany).

\section{Western blot}

The cell culture, toxin treatment, and western blot analysis were as described by Wang et al. (2019). After $24 \mathrm{~h}$ exposure to 1, 10, 100 and $1000 \mathrm{nM}$ MC-LR, the western blot analysis of Integrin $\beta 1$, FAK, Rho and ROCK was carried out.

\section{Statistical analysis}

Prism 8 statistical software (GraphPad Software, La Jolla, CA, USA) and IBM SPSS Statistics 22.0 (IBM Corp., Armonk, NY) were used for the statistical analysis. All the experimental data are from three independent experiments. The data shown are mean \pm standard deviation (SD) and differences with $p<$ 0.05 were considered to be statistically significant.

\section{Results}

\section{MC-LR inhibited vessel growth in aorta rings}

The rat aortic ring assay was used to verify that MC-LR inhibition of angiogenesis is a direct effect of MCLR. The aorta rings were embedded in Type I collagen and cultured in M199 medium containing 10\% FBS for microvascular endothelial cells to generate branching microvessels (Fig. 1a). Compared with the control group, the number of microvessels and migrated cells in 100 and $1000 \mathrm{nM} \mathrm{MC}$-LR-treated groups decreased significantly (Fig. 1b and c). No significant difference in cell transfer distance was observed between $100 \mathrm{nM}$ MC-LR-treated group and the control group, while the transfer distance of cell in 1000 nM MC-LR-treated group reduced significantly, which was $56.67 \%$ of the control group (Fig. 1 d). The findings showed that the sprouting angiogenesis from rat aorta was significantly blocked by MC-LR. 


\section{MC-LR inhibited vasculogenesis in zebrafish embryos}

To investigate the effects of MC-LR on zebrafish vasculogenesis, Tg (flk1: EGFP) embryos at $2 \mathrm{hpf}$ were exposed to MC-LR. As shown showed in Fig. 2, compared with the control group, the dorsal aorta (DA) in 100 and $1000 \mathrm{nM} \mathrm{MC}$-LR-treated groups at $24 \mathrm{hpf}$ has showed rougher appearance and curved shape, a curved posterior cardinal vein (PCV) has been observed accompanying growth inhibition, and the midcerebral veins (MceV) was suppressed. Moreover, The developmental absence of intersegmental vessel (ISV) has also been observed. Statistical analysis showed that ISV sprouting in $1000 \mathrm{nM}$ MC-LRtreated group was significantly blocked (Fig. 2b). The results showed that MC-LR blocked vasculogenesis in zebrafish embryos.

\section{MC-LR inhibited EC migration in zebrafish}

After exposing to MC-LR, the number of migrating ECs was quantified at $72 \mathrm{hpf}$. The percentage of EC migration from CHT was significantly dropped to $94.26 \%$ and $73.87 \%$ at the concentrations of 100 and $1000 \mathrm{nM}$ MC-LR relative to the control, respectively, which showed that exposure to MC-LR markedly inhibited EC migration from CHT (Fig. 3).

\section{MC-LR inhibited tube formation of HUVECs}

To evaluate whether MC-LR was involved in the process of angiogenesis, a matrigel tube formation assay was performed. As shown showed in Fig. 4, compared with the control group, the number of enclosed lumen, the number of the common node of enclosed lumen, the circumference of enclosed lumen and total lumen area in 100 and $1000 \mathrm{nM} \mathrm{MC}$-LR-treated groups decreased significantly following $12 \mathrm{~h}$ of incubation. The results showed that MC-LR inhibited tube formation of HUVECs.

\section{MC-LR inhibited HUVECs migration}

The adverse effect of MC-LR on cell migration is shown in Fig. 5. After exposure of HUVECs to MC-LR at $37^{\circ} \mathrm{C}$ for 12 and $24 \mathrm{~h}$, the distance of HUVEC migration in 1, 10, 100 and $1000 \mathrm{nM}$ MC-LR-treated groups reduced significantly in a dose-dependent manner compared with the control group. Especially in the highest concentration of MC-LR (1000 nM) treatment group, the distance of cell migration is the shortest, which is only $41.62 \%$ of the control after being exposed to MC-LR for $24 \mathrm{~h}$ (Fig. $5 \mathrm{~b}$ ). Therefore, MC-LR could inhibit HUVEC migration in a dose- and time- dependent manner.

\section{MC-LR induced HUVEC cytoskeleton damage}

To determine whether MC-LR induced disruption of the endothelial actin myosin cytoskeleton, the changes in cytoskeleton were measured following 24 hours of MC-LR exposure. The HUVEC cytoskeleton were shown in Fig. 6. In the control group, the cells exhibited a normal and clear distribution of actin microfilaments. The nuclei were widely surrounded by highly organized microfilaments with normal morphology. However, In contrast to control group, the microfilaments morphology in MC-LR-treated groups changed obviously. Microfilaments were condensed around the nucleus. HUVECs exhibited 
apoptotic properties and had a hollow nucleus with condensed chromatin (Fig. 6a). The mean fluorescence intensity of HUVECs in MC-LR-treated groups also reduced significantly compared with the control group (Fig. 6b). These finding suggests that MC-LR induces the dysfunction of HUVEC cytoskeleton system.

\section{MC-LR downregulated expression of integrin $\beta 1$, FAK, Rho, and ROCK}

To verify whether MC-LR inhibits HUVEC migration by integrin-mediated FAK/ROCK signaling pathway, integrin $\beta 1$, FAK, Rho and ROCK were analyzed by western blot analysis (Fig. 7a). The data showed that the expression of integrin $\beta 1$, FAK, Rho and ROCK downregulated significantly by MC-LR in a dosedependent manner (Fig. 7b-e).

\section{Discussions}

MC-LR is reported to be a group of well-known hepatotoxic cyclic peptides and is the main culprit for giving rise to serious cellular damage, as evident by various MC-LR concentrations associated with DNA injury, cytoskeleton disruption, mitochondria dysfunction, endoplasmic reticulum disturbance and cell cycle deregulation, all of which can contribute to apoptosis/programmed cell death (Brzuzan et al. 2009; Zhou et al. 2015; Faltermann et al. 2016). A tube formation assay has been typically used to demonstrate the angiogenic activity of vascular ECs in vitro (Shao and Guo 2004). EC migration is an essential component of angiogenesis that requires a tight regulation of the contractile and non-contractile states of the cell (Lamalice et al. 2007). Previous research work has proved that angiogenesis exhibits a positive correlation with cell motility (Robinet et al. 2005). In the present study, MC-LR inhibited vasculogenesis in zebrafish embryos, sprouting angiogenesis from rat aorta and tube formation of HUVECs. MC-LR also markedly inhibited EC migration from $\mathrm{CHT}$ in zebrafish and HUVEC migration. Therefore, it is plausible that MC-LR can inhibit vasculogenesis and angiogenesis by inhibition EC migration, leading to structural and functional damage to the vascular system.

The cytoskeleton is a complex association of detergent-soluble fractions of the cytoplasm, which plays vital roles in cell motility and shape formation of a cell. The perturbation of the cytoskeleton is important in vascular pathophysiology, which could involve not only direct inhibition of cell motility but also possibly interfere with guidance mechanisms for triggering cell-cell interaction networks (Thomas and Advani 2006; Hu et al. 2017). The toxic effects of MC-LR on the cytoskeletal structures have been widely investigated (Wickstrom et al. 1995; Zhou et al. 2015). Some studies have shown that exposure of human, mouse, or rat hepatocytes to MC-LR causes the rearrangement or collapse of the three components of the cytoskeleton. Multiple cytoskeletal and cytoskeleton-associated proteins were affected by MC-LR (Zhou et al. 2015). MC-LR can induce aggregation and collapse of microfilaments and microtubules in CIK cells, and even loss of some cytoskeleton structure (Huang et al. 2015). A relatively short-term exposure to MC-LR induced ROS mediate cytoskeletal disruption in the liver of carp (Jiang et al. 2013). However, there has been little concentrate on the endothelial cytoskeletal effects of MC-LR. In our study, MC-LR induced the change of microfilaments morphology. HUVECs had a hollow nucleus with 
condensed chromatin and exhibited apoptotic properties. Microfilaments were condensed around the nucleus. Hence, our study showed that the inhibiting effect of MC-LR on EC migration attributes to the changes of cytoskeleton, which is essential to angiogenesis.

We also investigated the mechanism of cytoskeletal disruption induced by MC-LR. Adhesion, migration, and invasion are important for HUVEC metastasis. The cytoskeleton confers the extent of integrin junction. The key part of the adhesive function of integrins is the ability that connects to the actin cytoskeleton (Delon and Brown 2007). The integrin $\beta 1$ and FAK is important for the focal adhesion complex formation, which controls cell adhesion and migration in human glioblastoma (Piao et al. 2009). The focal adhesion complex is directly related to ROCK pathways (Schofield and Bernard 2013). The Rho family of small monomeric GTPases is the monomeric GTP-binding protein, which contains Rho and Rac and is related to an abundance of cellular events, including cell cycle progression, cytoskeletal structure, and cell motility. It has been proved that ROCK stimulates actin myosin contractility and focal adhesion heavily involved in cell migration (Zohrabian et al. 2009). Integrin $\beta 1$-mediated FAK-Rho-ROCK-myosin signaling transduction pathway plays an important role in cell shape change, adhesion and migration (Hu et al. 2017). In the present study, western blot analysis showed that MC-LR down-regulated expressions of integrin $\beta 1$, FAK, Rho and ROCK in a dose-dependent manner, which suggested that MC-LR could induce cytoskeletal disruption, leading to the inhibition of EC migration by integrin-mediated FAK/ROCK signaling pathway.

\section{Conclusions}

In summary, the present study demonstrated that MC-LR blocked vasculogenesis in zebrafish embryos, sprouting angiogenesis from rat aorta and tube formation of HUVECs. MC-LR also markedly inhibited EC migration from CHT in zebrafish embryos and HUVEC migration. Therefore, the effect of MC-LR on vascular system was attributed to the inhibition of EC migration. MC-LR could also induce the disruption of cytoskeleton by integrin $\beta 1$-mediated FAK/ROCK signaling pathway. Taken together, MC-LR could inhibit EC migration by inducing disruption of cytoskeleton via integrin-mediated FAK/ROCK signaling pathway, leading to the inhibition of vasculogenesis and angiogenesis. Therefore, it is plausible that MCLR holds great adverse effect on vascular phylogeny. Our study helps to understand MC-LR risk to human cardiovascular health.

\section{Declarations}

Ethics approval and consent to participate We all declare that manuscript reporting studies do not involve any human participants, human data, or human tissue.

Consent for publication All authors approved the final manuscript as submitted for publication.

Competing interest The authors declare no conflict of interest. 


\section{Authors' contributions}

Tingzhang Hu conceived and designed the study. Qilong Wang was involved in methodology. Guoliang Chen contributed to original draft preparation. Qian Zhang and Mingxing Wang were associated with review and editing. Guixue Wang and Tingzhang Hu provided supervision. All authors have read and approved the final manuscript.

\section{Acknowledgements}

This study was supported by the Basic and Advanced Research Project of Chongqing (cstc2018jcyjAX0665) and the Public Experiment Center of State Bioindustrial Base (Chongqing), China.

\section{Data availability}

The datasets used and/or analyzed during the current study are available from the corresponding author on reasonable request.

\section{References}

Benson CE, Southgate L (2021) Angiogenesis. Epub ahead of print. https://doi.org/10.1007/s10456-02109768-8

Blacher S, Devy L, Burbridge MF, Roland G, Tucker G, Noël A, Foidart JM (2001) Improved quantification of angiogenesis in the rat aortic ring assay. Angiogenesis 4:133-142.

https://doi.org/10.1023/a:1012251229631

Brzuzan P, Woźny M, Ciesielski S, Łuczyński MK, Góra M, Kuźmiński H, Dobosz S (2009) Microcystin-LR induced apoptosis and mRNA expression of p53 and cdkn1a in liver of whitefish (Coregonus lavaretus, L.). Toxicon 54:170-183. https://doi.org/10.1016/j.toxicon.2009.03.032

Carmeliet P (2005) Angiogenesis in life, disease and medicine. Nature 438, 932-936. https://doi.org/10.1038/nature04478

Carmeliet P, Jain RK (2011) Molecular mechanisms and clinical applications of angiogenesis. Nature 473:298-307. https://doi.org/10.1038/nature10144

Chung AS, Ferrara N (2011) Developmental and pathological angiogenesis. Annu. Rev. Cell Dev Biol 27:563-584. https://doi.org/10.1146/annurev-cellbio-092910-154002

Davis GE, Camarillo CW (1995) Regulation of endothelial cell morphogenesis by integrins, mechanical forces and matrix guidance pathways. Exp Cell Res 216:1113-1123. 
Delon I, Brown NH (2007) Integrins and the actin cytoskeleton. Curr Opin Cell Biol 19:43-50. https://doi.org/10.1016/j.ceb.2006.12.013

Ding WX, Shen HM, Ong CN (2000) Microcystic cyanobacteria extract induces cytoskeletal disruption and intracellular glutathione alteration in hepatocytes. Environ Health Perspect 108:605-609.

https://doi.org/10.1289/ehp.00108605

Dziga D, Wasylewski M, Wladyka B, Nybom S, Meriluoto J (2013) Microbial degradation of microcystins. Chem Res Toxicol 26:841-852. https://doi.org/10.1021/tx4000045

Faltermann S, Grundler V, Gademann K, Pernthaler J, Fent K (2016) Comparative effects of nodularin and microcystin-LR in zebrafish: 2 . Uptake and molecular effects in eleuthero-embryos and adult liver with focus on endoplasmic reticulum stress. Aquat Toxicol 171:77-87.

https://doi.org/10.1016/j.aquatox.2015.12.001

Henty JL, Bledsoe SW, Khurana P, Meagher RB, Day B, Blanchoin L, Staiger CJ (2011) Arabidopsis actin depolymerizing factor4 modulates the stochastic dynamic behavior of actin filaments in the cortical array of epidermal cells. Plant Cell 23:3711-3726. https://doi.org/10.1105/tpc.111.090670

Hu T, Yang C, Fu M, Yang J, Du R, Ran X, Yin T, Wang G (2017) Cytotoxic effects of docetaxel as a candidate drug of drug-eluting stent on human umbilical vein endothelial cells and the signaling pathway of cell migration inhibition, adhesion delay and shape change. Regen Biomater 4:167-178. https://doi.org/10.1093/rb/rbx010

Huang X, Chen L, Liu W, Qiao Q, Wu K, Wen J, Huang C, Tang R, Zhang X (2015) Involvement of oxidative stress and cytoskeletal disruption in microcystin-induced apoptosis in CIK cells. Aquat Toxicol 165:41-50. https://doi.org/10.1016/j.aquatox.2015.05.009

Jiang J, Shan Z, Xu W, Wang X, Zhou J, Kong D, Xu J (2013) Microcystin-LR induced reactive oxygen species mediate cytoskeletal disruption and apoptosis of hepatocytes in Cyprinus carpio L. PLoS One 8:e84768. https://doi.org/10.1371/journal.pone.0084768

Kubis N, Levy BI (2003) Vasculogenesis and angiogenesis: molecular and cellular controls. Part 1: growth factors. Interv Neuroradiol 9:227-237. https://doi.org/10.1177/159101990300900301

Lamalice L, Boeuf FL, Huot J (2007) Endothelial cell migration during angiogenesis. Circ Res 100:782794. https://doi.org/10.1161/01.RES.0000259593.07661.1e

Liu H, Guo X, Liu L, Yan M, Li J, Hou S, Wan J, Feng L (2020) Simultaneous microcystin degradation and Microcystis aeruginosa inhibition with single enzyme Microcystinase A. Environ Sci Technol 54:88118820. https://doi.org/10.1021/acs.est.0c02155 
Nicosia RF, Ottinetti A (1990) Growth of microvessels in serum-free matrix culture of rat aorta. A quantitative assay of angiogenesis in vitro. Lab Invest 63:115-122

O'Neil JM, Davis TW, Burford MA, Gobler CJ (2012) The rise of harmful cyanobacteria blooms: the potential roles of eutrophication and climate change. Harmful Algae 14:313-334.

https://doi.org/10.1016/j.hal.2011.10.027

Piao Y, Lu L, de Groot J (2009) AMPA receptors promote perivascular glioma invasion via $\beta 1$ integrindependent adhesion to the extracellular matrix. Neuro Oncol 11:260-273.

https://doi.org/10.1215/15228517-2008-094

Preece EP, Hardy FJ, Moore BC, Bryan M (2017) A review of microcystin detections in estuarine and marine waters: environmental implications and human health risk. Harmful Algae 61:31-45. https://doi.org/10.1016/j.hal.2016.11.006

Preece EP, Hobbs W, Hardy FJ, O'Garro L, Frame E, Sweeney F (2021) Prevalence and persistence of microcystin in shoreline lake sediments and porewater, and associated potential for human health risk. Chemosphere 272:129581. https://doi.org/10.1016/j.chemosphere.2021.129581

Puddick J, Prinsep MR, Wood SA, Kaufononga SA, Cary SC, Hamilton DP (2014) High levels of structural diversity observed in microcystins from Microcystis CAWBG11 and characterization of six new microcystin congeners. Mar Drugs 12:5372-5395. https://doi.org/10.3390/md12115372

Robinet A, Fahem A, Cauchard JH, Huet E, Vincent L, Lorimier S, Antonicelli F, Soria C, Crepin M, Hornebeck W, Bellon G (2005) Elastin-derived peptides enhance angiogenesis by promoting endothelial cell migration and tubulogenesis through upregulation of MT1-MMP. J Cell Sci 118:343-356. https://doi.org/10.1242/jcs.01613

Schofield AV, Bernard O (2013) Rho-associated coiled-coil kinase (ROCK) signaling and disease. Crit. Rev. Biochem. Mol Biol 48:301-316. https://doi.org/10.3109/10409238.2013.786671.

Schwarzbauer JE (1997) Cell migration: may the force be with you. Curr Biol 7:292-294. https://doi.org/10.1016/s0960-9822(06)00140-0

Semenza GL (2007) Vasculogenesis, angiogenesis, and arteriogenesis: mechanisms of blood vessel formation and remodeling. J Cell Biochem 102:840-847. https://doi.org/10.1002/jcb.21523

Shao R, Guo X (2004) Human microvascular endothelial cells immortalized with human telomerase catalytic protein: a model for the study of in vitro angiogenesis. Biochem Biophys Res Commun 321:788794. https://doi.org/10.1016/j.bbrc.2004.07.033

Shi J, Deng H, Pan H, Xu Y, Zhang M (2017) Epigallocatechin-3-gallate attenuates microcystin-LR induced oxidative stress and inflammation in human umbilical vein endothelial cells. Chemosphere 168:25-31. https://doi.org/10.1016/j.chemosphere.2016.10.037 
Thomas TH, Advani A (2006) Inflammation in cardiovascular disease and regulation of the actin cytoskeleton in inflammatory cells: the actin cytoskeleton as a target. Cardiovasc Hematol Agents Med Chem 4:165-182. https://doi.org/10.2174/187152506776369926

Wang L, Chen G, Xiao G, Han L, Wang Q, Hu T (2020) Cylindrospermopsin induces abnormal vascular development through impairing cytoskeleton and promoting vascular endothelial cell apoptosis by the Rho/ROCK signaling pathway. Environ Res 183:109236. https://doi.org/10.1016/j.envres.2020

Wang Q, Liu Y, Guo J, Lin S, Wang Y, Yin T, Gregersen H, Hu T, Wang G (2019) Microcystin-LR induces angiodysplasia and vascular dysfunction through promoting cell apoptosis by the mitochondrial signaling pathway. Chemosphere 218:438-448. https://doi.org/10.1016/j.chemosphere.2018.11.019

Wickstrom ML, Khan SA, Haschek WM, Wyman JF, Eriksson JE, Schaeffer DJ, Beasley VR (1995) Alterations in microtubules, intermediate filaments, and microfilaments induced by microcystin-LR in cultured cells. Toxicol Pathol 23:326-337. https://doi.org/10.1177/019262339502300309

Zhang S, Du X, Liu H, Losiewic MD, Chen X, Ma Y, Wang R, Tian Z, Shi L, Guo H, Zhang H (2021) The latest advances in the reproductive toxicity of microcystin-LR. Environ Res 192:110254. https://doi.org/10.1016/j.envres.2020.110254

Zhou M, Tu WW, Xu J (2015) Mechanisms of microcystin-LR-induced cytoskeletal disruption in animal cells. Toxicon 101:92-100. https://doi.org/10.1016/j.toxicon.2015.05.005

Zhou S, Yu Y, Zhang W, Meng X, Luo J, Deng L, Shi Z, Crittenden J (2018) Oxidation of microcystin-LR via activation of peroxymonosulfate using ascorbic acid: kinetic modeling and toxicity assessment. Environ Sci Technol 52:4305-4312. https://doi.org/10.1021/acs.est.7b06560

Zohrabian VM, Forzani B, Chau Z, Murali R, Jhanwar-Uniyal M (2009) Rho/ROCK and MAPK signaling pathways are involved in glioblastoma cell migration and proliferation. Anticancer Res 29:119-123

\section{Figures}



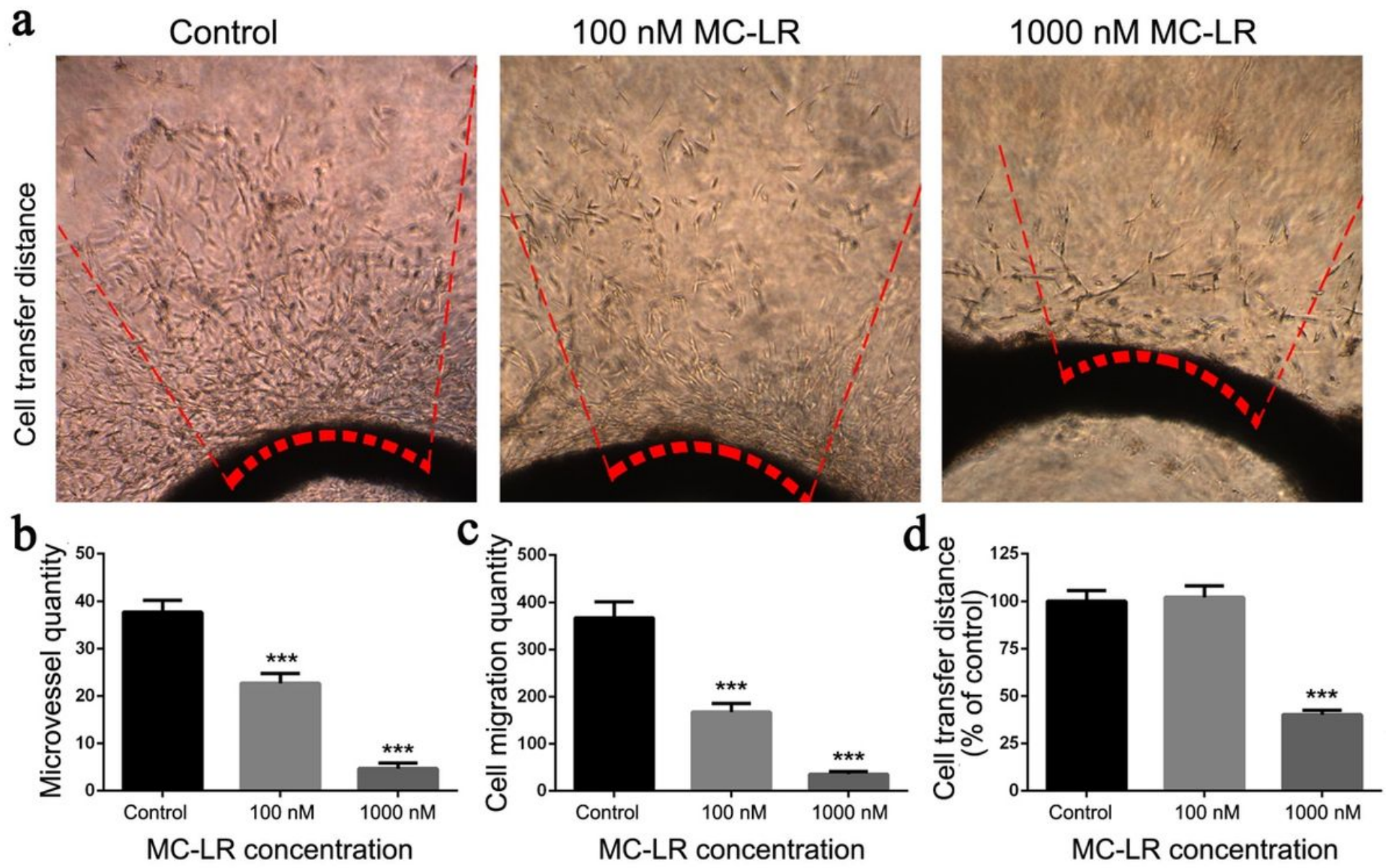

Figure 1

MC-LR inhibits sprouting angiogenesis in aorta rings. (a) Aortic ring assay. (b) Microvessel quantity. (c) Cell migration quantity. (d) Cell transfer distance. Aorta rings were treated with MC-LR for 2 weeks. The dotted line in Fig. $1 \mathrm{a}$ is the statistical calculation area. All values are represented as mean \pm SD of three replicates $(n=3)$. ***, $p<0.001$ vs control. 


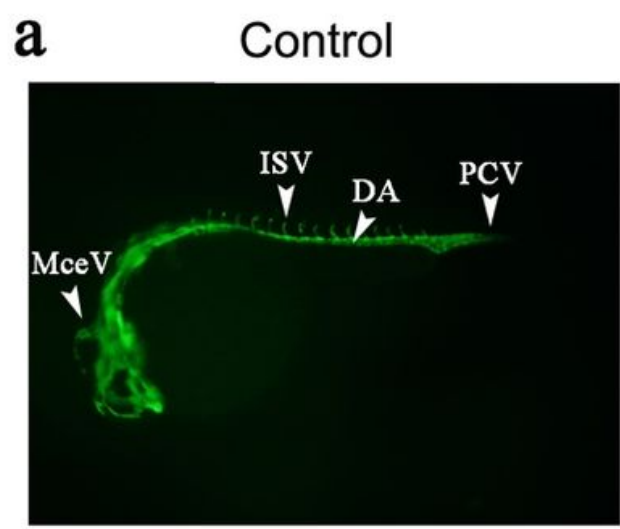

100 nM MC-LR
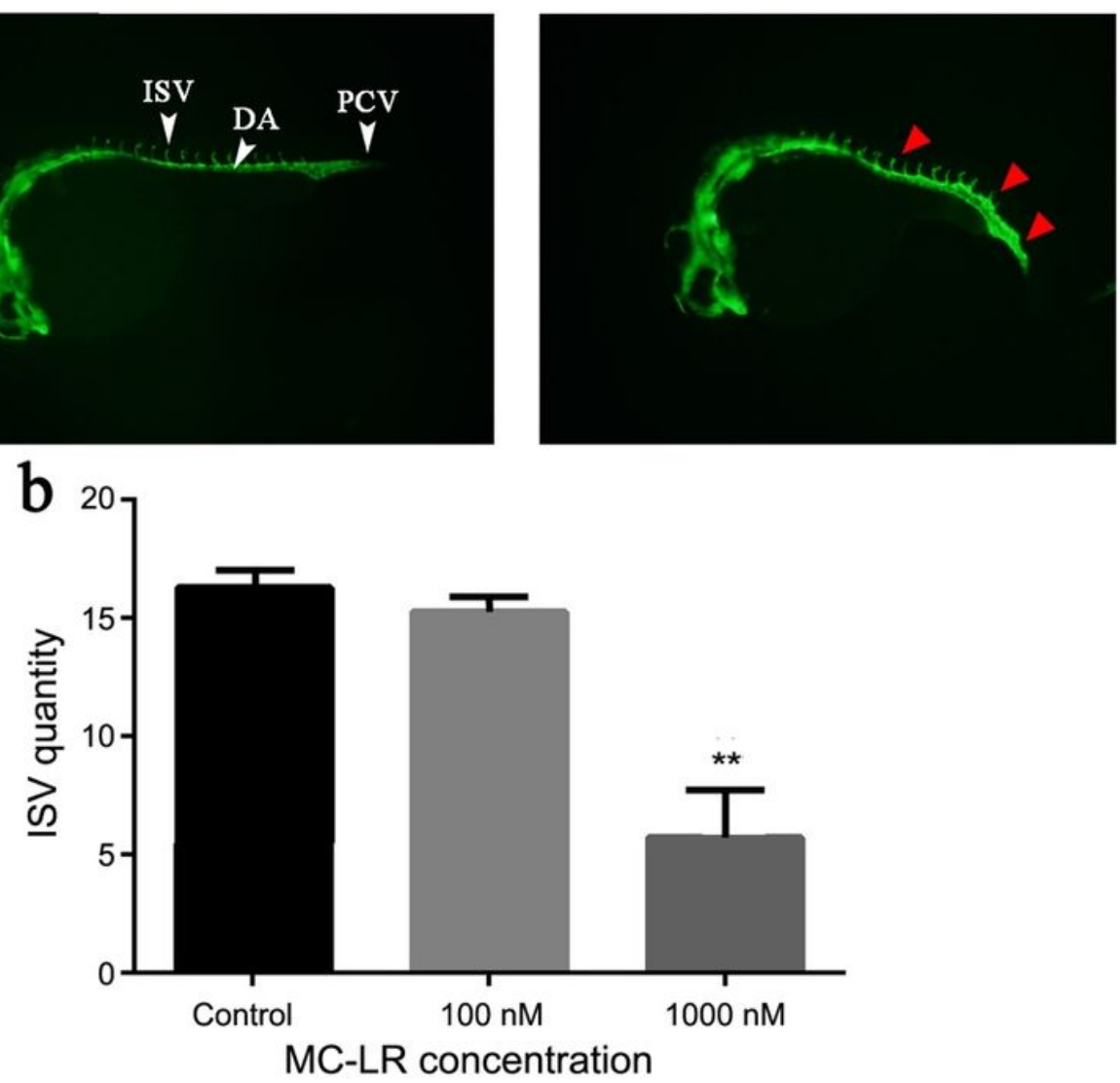

\section{Figure 2}

MC-LR inhibits vasculogenesis in zebrafish embryos. (a) The phenotype of vessels at $24 \mathrm{hpf}$. (b) ISV statistics based on Fig. 2A. Arrows in 100 and 1000 nM MC-LR-treated groups indicate vascular deletion. All values are represented as mean $\pm S D$ of three replicates $\left(n=3,30\right.$ embryos larvae/replicate). ${ }^{*}, p<$ 0.01 vs control. 


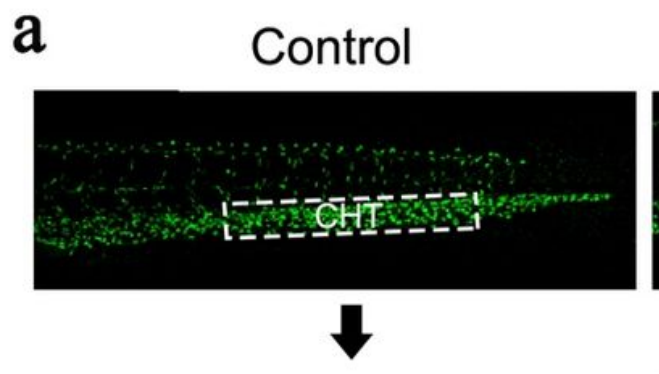

$100 \mathrm{nM}$ MC-LR
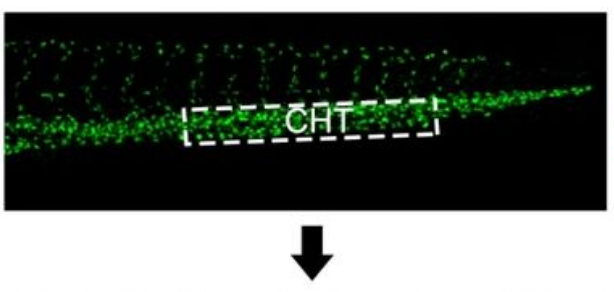

arishow

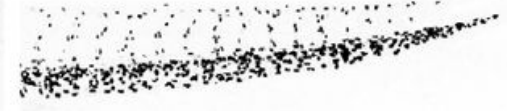

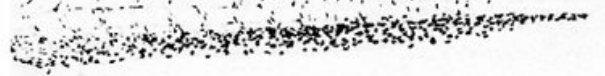

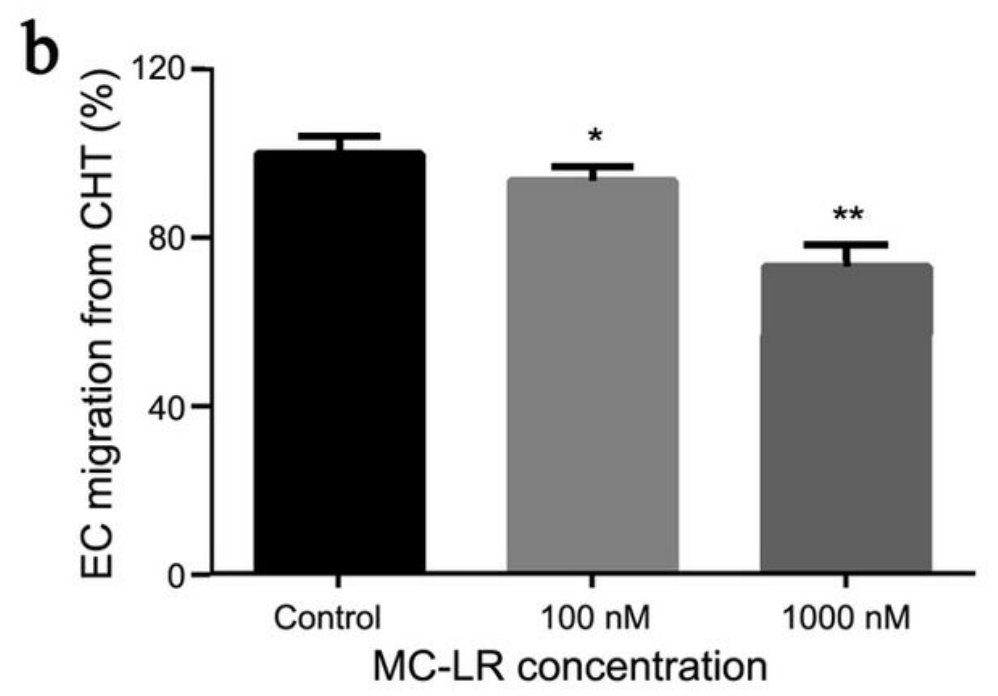

Figure 3

Effects of MC-LR on EC migration. (a) EC migration from CHT at $72 \mathrm{hpf}$. (b) The statistics of the cell migration from $\mathrm{CHT}$ based on Fig. 3a. All values are represented as mean \pm SD of three replicates $(\mathrm{n}=3)$. $*, p<0.05$ and $* *, p<0.01$ vs control. 
a

Control

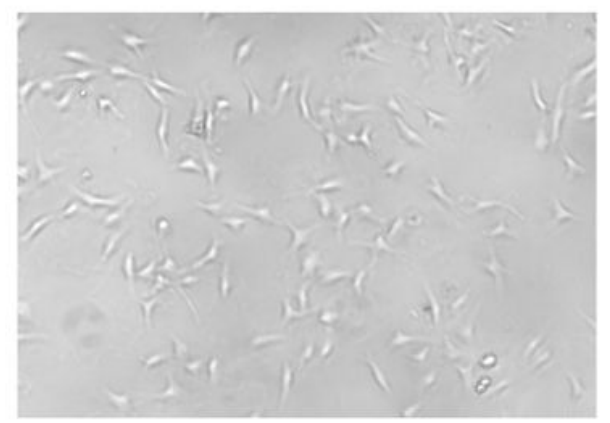

b

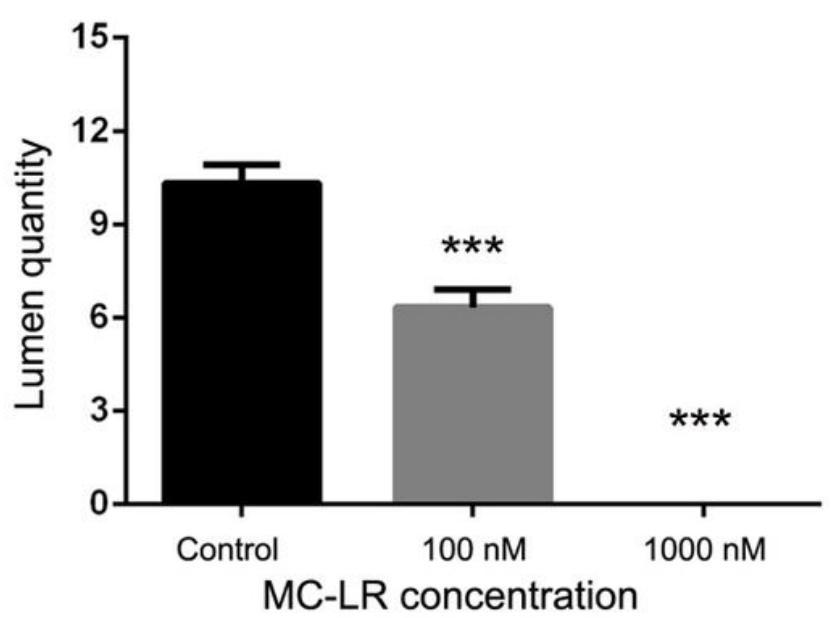

d

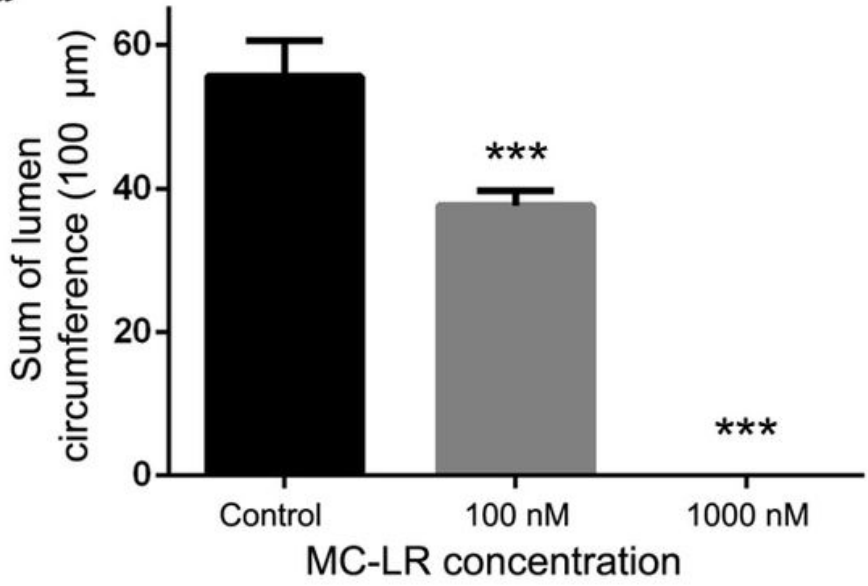

$1000 \mathrm{nM}$ MC-LR
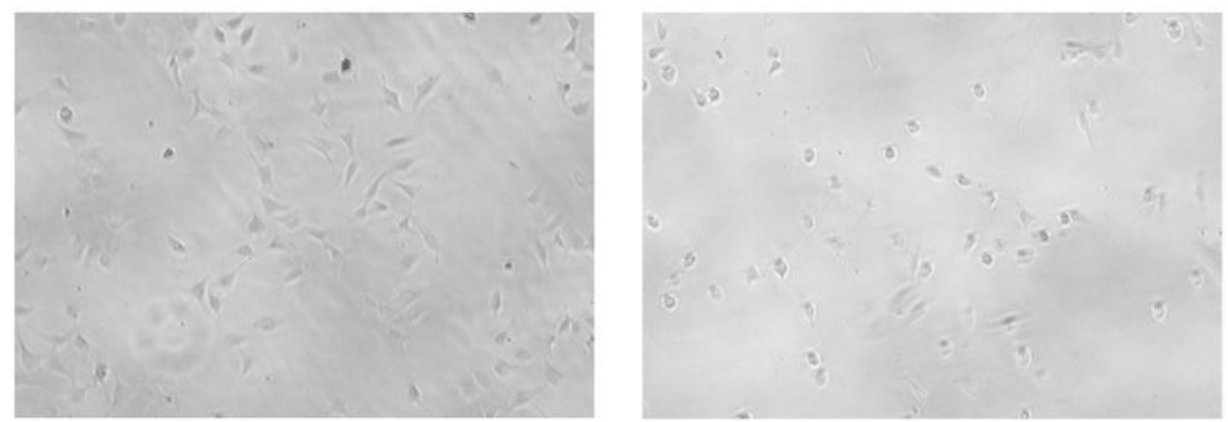

C

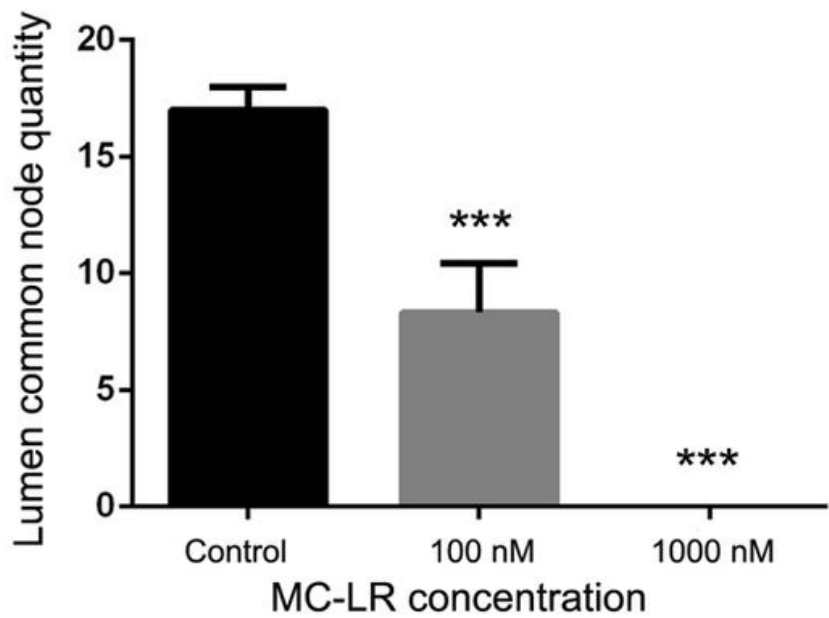

e

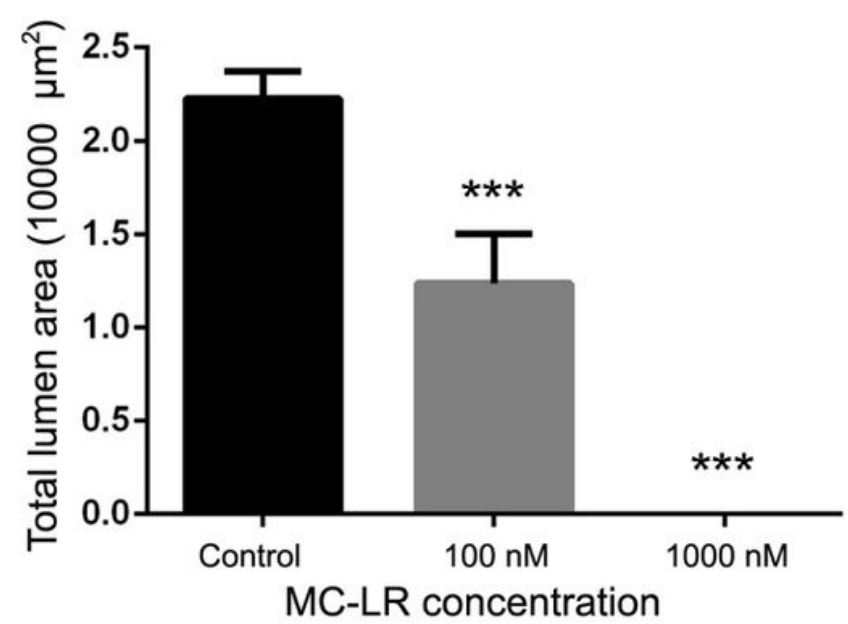

Figure 4

MC-LR inhibition of angiogenesis. (a) EC tube formation assay. (b) The number of enclosed lumen. (c) The number of the common node of enclosed lumen. (d) The circumference of enclosed lumen. (e) Total lumen area. All values are represented as mean \pm SD of three replicates $(n=3) . * \star *, p<0.001$ vs control. 

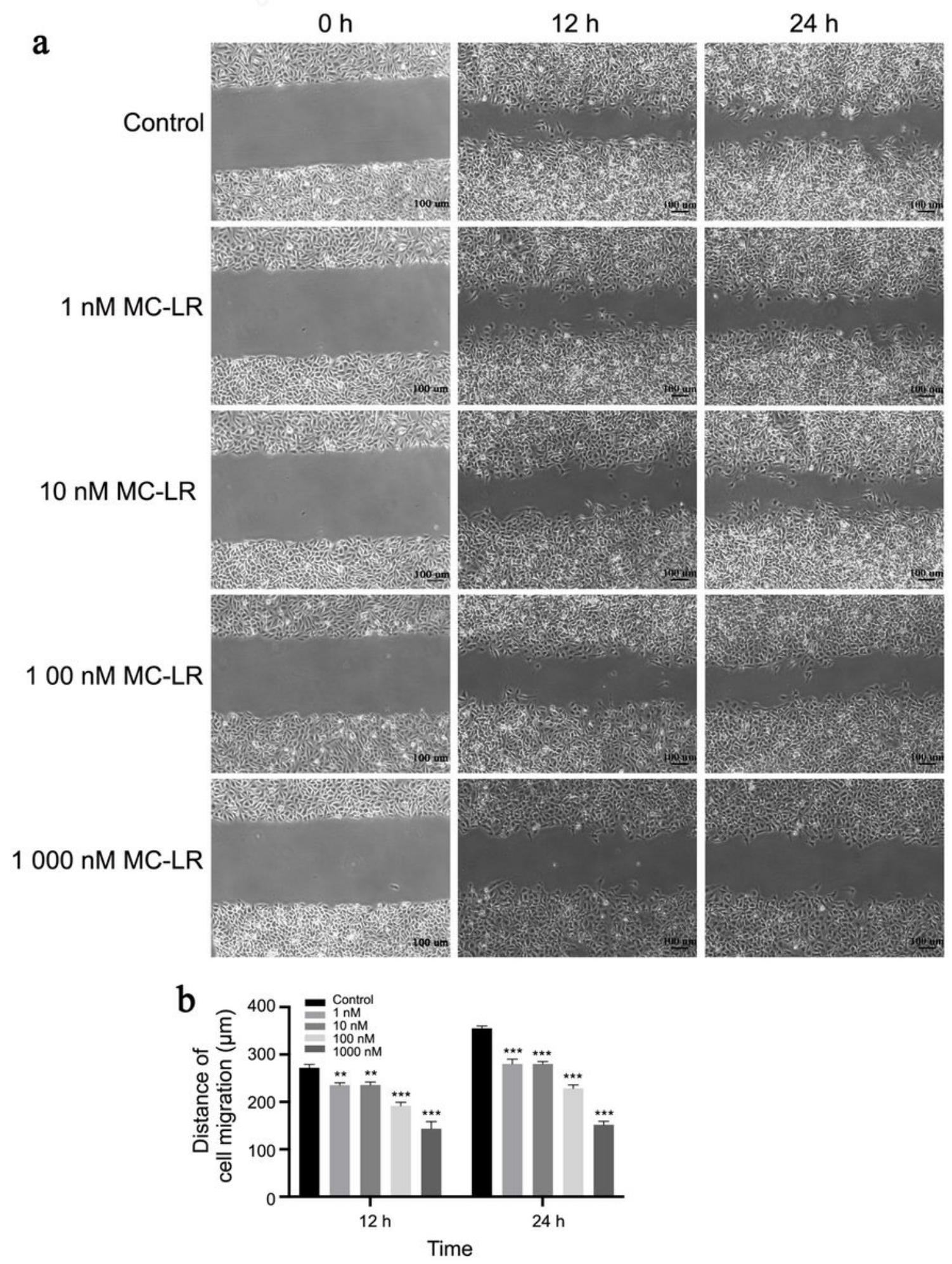

Figure 5

MC-LR inhibited HUVEC migration. (a) The microscope images of cell migration. (b) The distance of cell migration. All values are represented as mean \pm SD of three replicates $(n=3) . \star \star, p<0.01, \star \star \star, p<0.001$ vs control. 
a
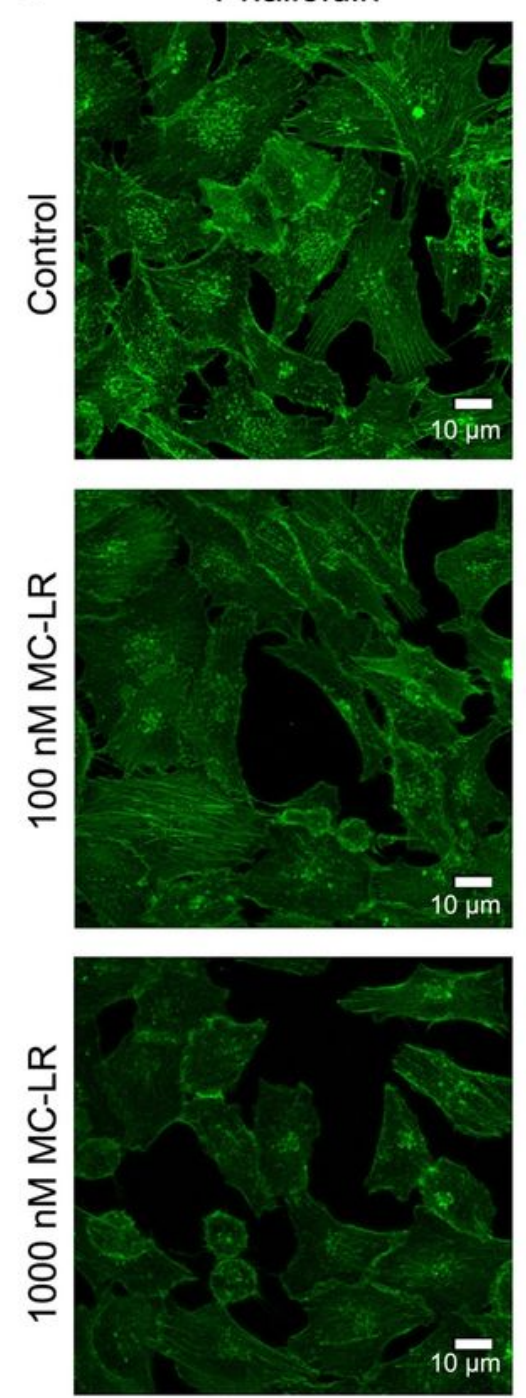

b

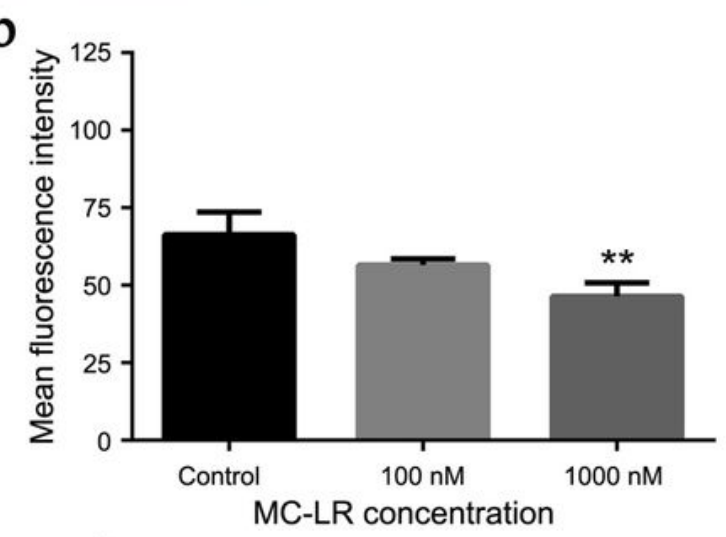

\section{Figure 6}

MC-LR induced actin myosin cytoskeleton reorganization. (a) Immunofluorescence images of MC-LR cytoskeleton. (b) quantitative analysis based on Fig. 6a. HUVECs were treated with MC-LR for $24 \mathrm{~h}$. All values are represented as mean \pm SD of three replicates $(n=3) .{ }^{\star *}, p<0.01$ vs control. 

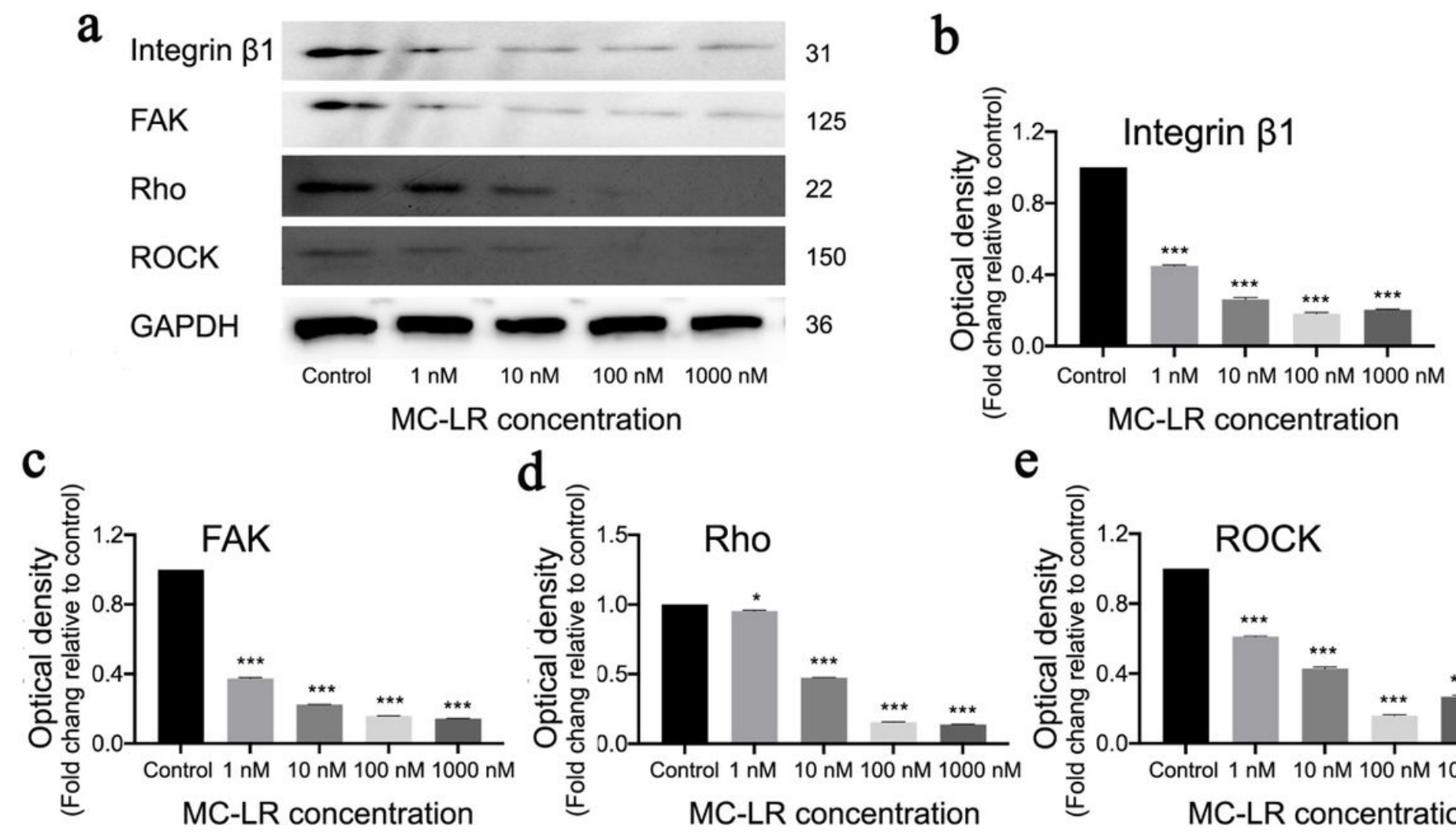

\section{Figure 7}

Western blot analysis of integrin $\beta 1, F A K$, Rho and ROCK in HUVECs. (a) Western blot. (b-e) The quantitative analysis based on Fig. 7a. HUVECs were treated with MC-LR for $24 \mathrm{~h}$. The vertical value in Fig. $7 \mathrm{~b}$-e is ratio of gray value of target protein / gray value of internal reference protein (GAPDH) based on Fig. 7A. All values are represented as mean \pm SD of three replicates $(n=3) .{ }^{*}, p<0.05,{ }^{\star \star \star}, p<0.001$ vs control.

\section{Supplementary Files}

This is a list of supplementary files associated with this preprint. Click to download.

- GraphicalAbstract.tif 